http://jmscr.igmpublication.org/home/ ISSN (e)-2347-176x ISSN (p) 2455-0450 crossref DOI: https://dx.doi.org/10.18535/jmscr/v7i9.79

\title{
Carotid Doppler as a Screening Tool for Diagnosis of Impending Stroke in Patients with Metabolic Syndrome
}

\author{
Authors \\ Dr Vaja Mukhtarahmed, Dr S.A. Kanitkar, Dr Anu Gaikwad, Dr Nuvvula Siva \\ Krishna, Dr George Thomson Ben, Dr Dumpala Rajneesh Reddy, Dr Sura Sreekar \\ Department" of "General Medicine" Dr. D. Y. "Patil Medical College, Hospital and Research Centre, \\ Pimpri, Pune \\ *Corresponding Author \\ Dr Vaja Mukhtarahmed \\ C1-606 Mahindra Antheia, Nehrunagar, Pimpri, Pune-411018, India
}

\begin{abstract}
Background: Metabolic syndrome and its individual criteria poses an increased risk for atherosclerotic diseases. Metabolic syndrome is also associated with increase in carotid intima-media thickness, which is a marker of preclinical atherosclerosis. Hence this study was aimed to determine to find out correlation between metabolic syndrome and increase in carotid intimal media thickness.

Methods: All patients with stroke age more 30 years admitted in Department of General Medicine during the study period from May 2017 to April 2020 were included in study. A total of 50 patients presenting with stroke were assessed and were classified as metabolic syndrome or non-metabolic syndrome based on IDF criteria who fulfilled the selection criteria during the study period were enrolled.

Results: Out of 50 stroke patients studied, 29 patients (17 males and 12 females) had metabolic syndrome according to IDF criterion. Occurrence of metabolic syndrome in patients with stroke was $58 \%$. Out of total $29(58 \%)$ patients with metabolic syndrome, $17(58.6 \%)$ were male and $12(57.1 \%)$ patients were. Out of total patients in the study group, 29 patients had increased carotid intimal thickness out of which 21(72.4\%) patients had metabolic syndrome with 12(75\%) Males and 09(69.23\%) Females. It was found to be statistically significant with $p$ value 0.03 .

Conclusions: Accelerated rate of atherosclerosis in found in patients with metabolic syndrome and there is increase in CIMT in these patients, so carotid doppler can be used for predicting atherosclerotic changes in such patients in early stages.

Keywords: Atherosclerosis, Carotid intima media thickness, Metabolic syndrome, Carotid Doppler.
\end{abstract}

\section{Introduction}

Stroke is a sudden loss of neurologic function (global or focal) due to vascular disruption (infarction or hemorrhage) in central nervous system. ${ }^{1}$ Approximately 15 million acute onset stroke events occur worldwide every year. Two third of them live in countries like India which are low to middle income countries. It has been anticipated that by year 2050, $80 \%$ of stroke events will occur in people of these regions. Stroke is the leading cause of death and disability in developing countries and average age of 
presentation of stroke for these patients is much younger than in developed countries and is almost fifteen years younger than developed countries. Metabolic syndrome has attracted tremendous attention in recent years and $\geq 5$ definitions has been proposed for metabolic syndrome by different medical societies. Prevalence of metabolic syndrome in developed countries is around 25-40\% and India has strikingly high prevalence when compared with rest of Asia. It has now been recognized as a highly prevalent problem in many other countries worldwide. ${ }^{2}$ Presence of metabolic syndrome in a individual has a major risk for development of both atherosclerosis and type 2 diabetes mellitus and its prevalence increases to two to three times higher in individuals with metabolic syndrome than in age-matched controls. ${ }^{3}$ In addition, metabolic syndrome is also associated with increase in carotid intima-media thickness (CIMT), which is a marker of preclinical atherosclerosis. It can predict future neurological and cardiovascular morbidity and mortality, specifically stroke and myocardial infarction respectively. ${ }^{4}$ Many studies have shown, increased carotid intima media thickness (CIMT) as a good indicator of atherosclerosis. It has also been reported to correlate with peripheral artery disease. ${ }^{4}$ It includes the abdominal obesity, dyslipidemia, increased blood pressure and insulin resistance. These components are independent risk factors for stroke. There have been many evidences that metabolic syndrome is an important risk factors for promoting atherosclerotic diseases. This association boosts the clinical use of the metabolic syndrome in the identification of individuals who are at a higher risk of suffering a stroke. Abdominal adiposity and insulin resistance appear play a central role in the pathogenesis of this syndrome and its individual components. ${ }^{5}$ Many inflammatory mediators are released in circulation in presence of visceral obesity of which important ones are tumour necrosis factor, leptin, interleukin-6 and angiotensinogen which is produced by the adipose tissue which suggests an crucial role of visceral obesity in metabolic syndrome. ${ }^{6}$ Tumor Necrosis Factor affects insulin signalling by inhibiting it and thus contributes to insulin resistance. Increase in blood pressure is due to Leptin which activates the immune system. Interlukin- 6 affects by stimulating the production of C-reactive protein in the liver in obese individuals. Angiotensinogen which is produced by adipose tissue is converted to angiotensin II which produces its adverse outcomes through angiotensin II type 1 receptor (AT1) which leads to secretion of aldosterone and its effects i.e. oxidative stress, sympathetic stimulation and vasoconstriction. ${ }^{6}$ It is also found that there is increased plasma levels of fibrinogen and coagulation factors like factor $7 \& 8$, which causes a potential hypercoagulable state in an individual along with increased plasminogen activator inhibitor-1 levels, leading to a hypofibrinolytic state. All these elements predispose to thrombosis and hence increase the probability of stroke and coronary artery disease. ${ }^{6}$ If the young adults and adolescents can be screened for presence of metabolic syndrome early and managed appropriately, the incidence of stroke could be reduced and thereby the morbidity. Hence the present study was undertaken to assess changes in carotid intima media thickness in patients with stroke with metabolic syndrome so as to find out whether increase in carotid intimal thickness can be used as marker for impending stroke in patient with metabolic syndrome.

\section{Methods}

All patients with stroke age more 30 years admitted in Department of General Medicine during the study period from May 2017 to April 2020 were included in study. A total of 50 patients presenting with stroke were assessed and were classified as metabolic syndrome or nonmetabolic syndrome based on IDF criteria who fulfilled the selection criteria during the study period were enrolled. 
The diagnosis of "metabolic syndrome" was made According to "International Diabetes Federation" Criteria:

1. Central obesity (In patients with waist circumference $\geq 90 \mathrm{~cm}$ for South Asian men and $\geq 80 \mathrm{~cm}$ for South Asian women). With any two of the following factors:

2. Triglycerides: $\geq 150 \mathrm{mg} / \mathrm{dl}$, or on any specific treatment for already known lipid abnormality.

3. HDL cholesterol: $\leq 40 \mathrm{mg} / \mathrm{dl}$ in male, $\leq 50$ $\mathrm{mg} / \mathrm{dl}$ in females, or on any specific treatment for already known lipid abnormality.

4. Blood Pressure: $\geq 130 / 85 \mathrm{~mm} \mathrm{Hg}$, or on any specific treatment for already known case of hypertension.

5. Fasting blood glucose: $\geq 100 \mathrm{mg} / \mathrm{dl}$, or on any specific treatment for already known case of type 2 diabetes mellitus.

\section{Inclusion Criteria}

1. All patient presenting with stroke and clinically diagnosed as stroke and confirmed with computerized topography (CT scan) or magnetic resonance imaging (MRI) as Ischemic stroke.

2. Age more than 30 .

3. Patients with at least one stroke episode.

4. Stroke duration of less than 2 weeks.

\section{Exclusion Criteria}

1. Patient diagnosed with Hemorrhagic stroke on CT scan or MRI scan.

2. Stroke because of other causes like vasculitis, metastatic tumors, post-seizure paresis and head trauma.

The ethical clearance was obtained from the Institutional Ethical committee.

\section{Procedure}

All the participants were also informed about the study procedure and the information required from them for the study. A voluntary informed written consent was taken from the participant those who consented were included in the study. A strict confidentiality was maintained about the personal details of the participants and information related to the study. Consent form was available in English, Hindi as well as in Marathi. In case of illiterate participants/relatives' consent was taken in presence of witness. Any third-party person not directly attached to the study was taken as witness. Detailed history including risk factors in the development of CVA, history of smoking \& alcohol abuse, history of stroke, family history of stroke, use of antidiabetic, antihypertensive and antihyperlipidemic drugs, clinical findings and investigation reports have been taken. All patients were thoroughly examined including all peripheral pulses as well as carotid examination for any evidence of atherosclerosis. A predesigned semistructured questionnaire was prepared based on the review of literature on metabolic syndrome and stroke. Waist and hip circumferences were measured, fundus was examined for every patient for any evidence of any hypertensive changes and papilledema. Cardiovascular, respiratory, abdominal and CNS examination was done. At least three blood pressure measurements with same standard mercury sphygmomanometer and Littman's stethoscope in supine position was taken. For all patients, blood pressure was taken after a rest period of 15 minutes, measured by a mercury sphygmomanometer with the patient in supine position in the upper limb not involved due to hemiparesis. The final blood pressure estimate was the average of 3 readings. Individuals already on medication for hypertension was considered as hypertensive patient even if the blood pressure levels during study was found to be normal. For diabetics and patients aged above 65; postural changes were checked by taking blood pressure immediately and two minutes after standing. Waist circumference was measured using a standard measuring tape, which was positioned in the mid-way between the lower rib and iliac crest. BMI was calculated for each patient. The fasting lipid profile (FLP) i.e. total cholesterol, TG, HDL, LDL was estimated using COBAS 311 using 
Roche reagents by approved enzymatic method after centrifugation followed by serum separation. $2 \mathrm{ml}$ fasting blood samples were used for the measurement of glucose level in the blood by spectrophometric GOD-POD method i.e. Glucose oxidase per oxidase method using COBAS 311 analyzer. The carotid intima media thickness was determined by imaging of bilateral common carotid artery using a high frequency Bmode ultrasonography with patients in supine position and head tilted backwards. After location of the carotid arteries, the probe was rotated $90^{\circ}$ to obtain the longitudinal image of the anterior and posterior walls. The maximum CIMT was measured for the common carotid artery, the internal carotid arteries and at the bifurcation and was expressed as a mean aggregate value. CIMT values of $>0.1 \mathrm{~cm}$ were considered abnormal and confer increased absolute risk of CHD. ${ }^{7}$,

\section{Statistical Analysis}

Data analysis was done using Microsoft excel sheet. Tables and graphs were prepared for the variables. Pearson chi-square test was used for categorical variables in the study. In our study $\mathrm{P}$ value less than 0.05 was considered to be significant. The continuous variables were assessed using students $\mathrm{T}$ test.

\section{Results}

This study was conducted among 50 cases of ischemic stroke and was evaluated for the various components of metabolic syndrome according to IDF criterion. Following tables and graphs shows observations of the study.

\section{Incidence of Metabolic Syndrome among the Study Population (n=50) IDF criterion}

\begin{tabular}{|l|c|c|}
\hline Prevalence (IDF) & No of subjects & Percentage \\
\hline Metabolic syndrome & 29 & $58 \%$ \\
\hline Without Metabolic syndrome & 21 & $42 \%$ \\
\hline
\end{tabular}

Prevalence of metabolic syndrome According to IDF criterion

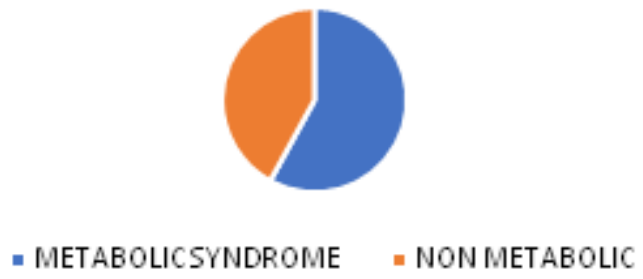

- Out of 50 stroke patients studied, 29 patients (17 males and 12 females) had metabolic syndrome according to IDF criterion. Occurrence of metabolic syndrome in patients with stroke was $58 \%$. Out of total $29(58 \%)$ patients with metabolic syndrome, $17(58.6 \%)$ were male and $12(57.1 \%)$ patients were.

- Majority of patients in our study were in age group between 51-60 with a total number of 21 patients, of which $9(42.9 \%)$ were males and $12(57.1 \%)$ were females. Second most common age group being 61 70 years with total 19 patients, with 13 male and 6 female patients i.e. $68.4 \%$ males \& $31.6 \%$ females.

- In our study number of male patients were more than females. 


\section{Component for Mets among study subjects}

\begin{tabular}{|c|c|c|}
\hline Component for Mets & Freq & Percentage \\
\hline Hypertension & 37 & $74 \%$ \\
\hline Diabetes & 34 & $68 \%$ \\
\hline Decreased HDL & 31 & $62 \%$ \\
\hline Raised TG & 27 & $54 \%$ \\
\hline Increased WC-IDF & 31 & $62 \%$ \\
\hline Increased WC-NCEP & 21 & $42 \%$ \\
\hline $\begin{array}{r}80 \\
60 \\
40 \\
20 \\
0\end{array}$ & $\alpha^{0}$ & \\
\hline
\end{tabular}

- Individual components of metabolic

common component being hypertension syndrome (as per IDF criterion) have been followed by diabetes mellitus and least shown in the above table with most common being raised triglycerides.

Various variables of metabolic syndrome and their mean values with sex wise distribution:

\begin{tabular}{|l|c|c|c|}
\hline VARIABLES & Males $(\mathbf{n}=\mathbf{2 9})$ & Female(n=21) & P value \\
\hline Mean HDL (mg/dl) & $51.07+15.87$ & $50.09+18.72$ & 0.845 \\
\hline Mean TGL (mg/dl) & $169.69+62.24$ & $174.14+51.57$ & 0.790 \\
\hline Mean FBG (mg/dl) & $117.66+39.89$ & $150.95+64.02$ & 0.028 \\
\hline Mean WC (inches) & $37.43+4.47$ & $36.49+5.76$ & 0.519 \\
\hline Mean BP (mmHg) & $149.24 / 96.07 \pm$ & $156.57 / 95.33 \pm$ & 0.293 \\
& $23.87 / 15.04$ & $24.28 / 10.66$ & \\
\hline Weight & $82.03+10.14$ & $83.92+10.67$ & 0.528 \\
\hline Height & $168.51+6.41$ & $169.11+8.19$ & 0.776 \\
\hline BMI & $29.00+4.40$ & $29.36+3.30$ & 0.755 \\
\hline
\end{tabular}

Table: Carotid Intimal Thickness with Metabolic Syndrome

\begin{tabular}{|c|c|c|c|c|}
\hline \multirow{2}{*}{$\begin{array}{l}\text { Carotid Intimal } \\
\text { Media Thickness }\end{array}$} & \multicolumn{3}{|c|}{ Metabolic syndrome } & \\
\hline & No & Yes & Total & \multirow{4}{*}{0.033} \\
\hline No & $13(61.9 \%)$ & $08(38.1 \%)$ & 21 & \\
\hline Yes & $08(27.6 \%)$ & $21(72.4 \%)$ & 29 & \\
\hline TOTAL & $21(42.00 \%)$ & $29(58.00 \%)$ & 50 & \\
\hline
\end{tabular}

Carotid Thickness


Metabolic syndrome and increased carotid intimal media thickness sex wise comparison

\begin{tabular}{|l|c|c|c|}
\hline $\begin{array}{l}\text { Carotid Intimal Media } \\
\text { Thickness }\end{array}$ & Male & Female & Total \\
\hline Mets & $12(75 \%)$ & $09(69.23 \%)$ & $21(72.4 \%)$ \\
\hline Without Mets & $04(25 \%)$ & $04(30.77 \%)$ & $08(27.6 \%)$ \\
\hline Total & 16 & 13 & 29 \\
\hline
\end{tabular}

*Figures in bracket show column percentage

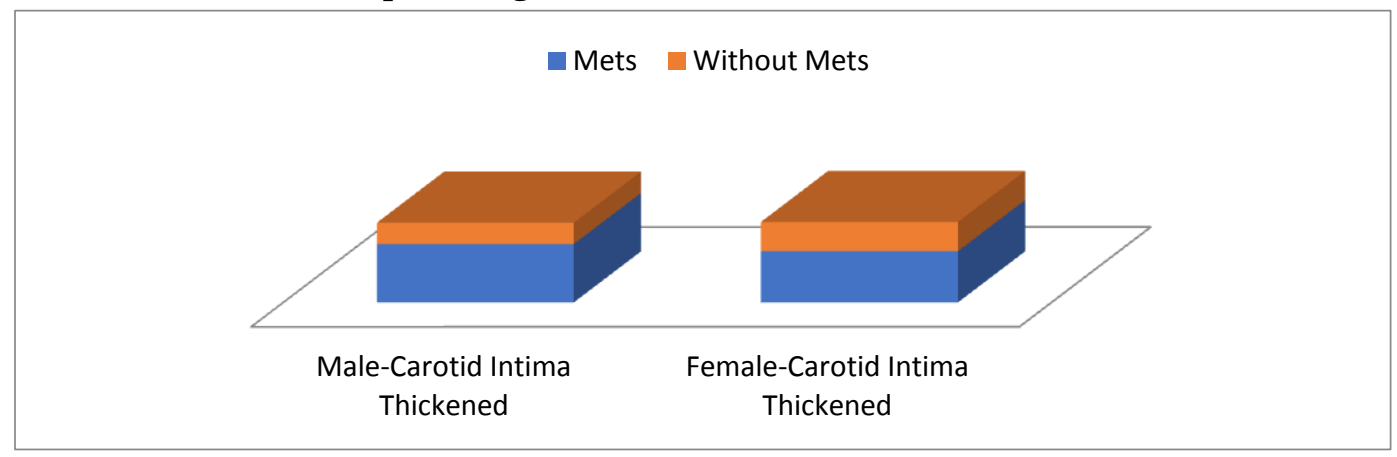

Out of total patients in the study group, 29 patients had increased carotid intimal thickness out of which $21(72.4 \%)$ patients had metabolic syndrome with 12(75\%) Males and 09(69.23\%) Females. It was found to be statistically significant with $\mathrm{p}$ value 0.03.It suggests that patients with metabolic syndrome has high risk of increase in carotid intimal media thickness and thus increasing chances of impending thrombotic stroke. Present study shows that carotid doppler is an important non-invasive diagnostic stool. It can be used in screening in high risk asymptomatic patients, and for determining treatment protocol. Every metabolic syndrome patient should be screened for increased carotid intimal media thickness and more intensive preventive management should be done to prevent stroke.

\section{Discussion}

In the present study, the Carotid Intimal Media Thickness were raised and suggest that, a considerable individual with metabolic syndrome are likely to have higher CIMT levels. In a study by Koskinen $\mathrm{J}$ et al, metabolic syndrome was significantly associated with accelerated CIMT progression. ${ }^{8}$ The mean CIMT noted in the present study corroborates with the result of the study by Timoteo AT et al, who reported mean CIMT as $0.88 \pm 0.33 \mathrm{cms} .{ }^{9}$ In the European Lacidipine Study on Atherosclerosis (ELSA), metabolic syndrome was associated in a bivariate model with a 4year change in CIMT among 1734 hypertensive subjects aged 45 to 75 years at baseline. ${ }^{10}$ Ispessimento Medio Intimale e Rischio cardiovascol are media-intima thickness and cardiovascular risk] (ISMIR) study, showed an association between metabolic syndrome and CIMT. ${ }^{12}$ The prevalence of an increased CIMT (defined as common carotid artery CIMT $>0.80 \mathrm{cms}$ ) was significantly higher in patients with metabolic syndrome than in patients without it, and mean CIMT was also significantly higher. Increased CIMT is reported to be associated with stroke in subjects including those with diabetes. ${ }^{11,12}$ Eguchi et al $^{13}$ too supported these findings while Johnsen et al ${ }^{14}$ was of the view that CIMT was associated with hypertensive vascular disease. Overall in nutshell, a considerable subset of patients with metabolic syndrome are likely to have raised CIMT. However, the rise in CIMT among the patients with metabolic syndrome was independent of the number of components of metabolic syndrome criteria. These finding need further validations due to potential limitations of this study, namely single centre study, smaller sample size, smaller subset of female participation, age distribution was not uniform, majority of the patients had all five abnormalities and almost all the patients presented with history of hypertension and diabetes mellitus whose 
duration of disease was not considered in the assessment. The treatment history and treatment compliance were not analyzed.

\section{Conclusion}

From the present study it can be concluded that there is accelerated rate of atherosclerosis in patients with metabolic syndrome and there is increase in Carotid Intimal Media Thickness in patients with metabolic syndrome, so carotid doppler can be used for predicting atherosclerotic changes in such patients. Carotid doppler is a noninvasive, easily available and cheap method of investigation to assess atherosclerosis. More intensive preventive management can be done to prevent neurological morbidity and mortality. Thus, it should be used as a first line investigation in asymptomatic patient with metabolic syndrome to prevent dreaded complication of stroke.

\section{References}

1. Elkind SVM, Sacco RL. Pathogenesis, Classification and Epidemiology of Cerebrovascular Disease. Merritt's Neurology. Section IV. Chapter 37.12thed. Lewis $\mathrm{P}$ R, Timothy A P. Lippincott Williams \& Wilkins 2010 ; 217-229.

2. Alberti KG, Zimmet P, Shaw J. The metabolic syndrome - a new world-wide definition. Lancet. 2005 Sep 2430;366(9491):1059-62.

3. Crouse III JR, Tang R, Espeland MA, Terry JG, Morgan T, Mercuri M. Associations of extracranial carotid atherosclerosis progression with coronary status and risk factors in patients with and without coronary artery disease. Circulation. 2002 Oct 15;106(16):2061-6.

4. Kuller L, Borthani N, Furberg C, Gardin J, Manolio T, O'leary D, et al. Prevalence of subclinical atherosclerosis and cardiovascular disease and association with risk factors in the Cardiovascular Health Study. Am J Epidemiol. 1994 Jun 15;139(12):1164-79.
5. Francesco D, Erica R, Walter A. The metabolic syndrome and the risk of thrombosis. Haematol. 2007;92:297-9.

6. Grundy SM. Definition of metabolic syndrome, Report of the National Heart, Lung, and Blood Institute/American Heart Association conference on scientific issues related to definition. Circ. 2004;109:433- 8.

7. O'Leary DH, Bots ML. Imaging of atherosclerosis: Carotid intima-media thickness. Eur Heart J. 2010 Jul;31(14):1682-9.

8. Koskinen J, Kahonen M, Viikari JS, Taittonen L. Conventional CV risk factors and MetS in predicting carotid intimamedia thickness progression in young adults. The CV risk in young Finns study. Circulation. 2009;120:229-36.

9. Timóteo AT, Carmo MM, Ferreira RC. Can metabolic syndrome presence predict carotid intimamedia thickness? J Clin Hypertens (Greenwich). 2012;14(8):50713.

10. Zanchetti A, Hennig M, Baurecht H, Tang R, Cuspidi C, Carugo S, et al. Prevalence and incidence of the metabolic syndrome in the European Lacidipine Study on Atherosclerosis (ELSA) and its relation with carotid intima-media thickness. Journal of hypertension. 2007 Dec 1;25(12):2463-70.

11. O'Leary DH, Polak JF, Kronmal RA, Manolio TA, Burke GL, Wolfson SK Jr: Carotid-artery intima and media thickness as a risk factor for myocardial infarction and stroke in older adults. Cardiovascular Health Study Collaborative Research Group. N Engl J Med 340:14- 22,199.

12. Koshiyama H, Shimono D, Kuwamura N, Minamikawa J, Nakamura Y: Rapid communication: inhibitory effect of pioglitazone on carotid arterial wall thickness in type 2 diabetes. J Clin Endocrinol Metab2001;86:3452-3456. 
13. Eguchi K, Kario K, Shimada K: Greater impact of coexistence of hypertension and diabetes on silent cerebral infarcts. Stroke 2003;34:2471-2474.

14. Johnsen SH, Mathiesen EB: Carotid plaque compared with intimamedia thickness as a predictor of coronary and cerebrovascular disease. CurrCardiol Rep 2009;11:21-27.
Abbreviations
CIMT: Carotid Intima-Media Thickness
IDF: International Diabetes Federation
HDL: High Density Lipoprotein
CT scan: Computerized Topography
MRI: Magnetic Resonance Imaging
CVA: Cerebrovascular Accident
CNS: Central Nervous System
TG: Triglyceride
LDL: Low Density Lipoprotein
CHD: Coronary Heart Disease
Mets: Metabolic Syndrome
WC: Waist Circumference
NCEP: National Cholesterol Education
Programme
FBG: Fasting Blood Glucose
BMI: Body Mass Index 